\title{
A COMPARATIVE STUDY OF THERMODYNAMIC PROPERTIES OF STRUCTURALLY RELATED PHENOTHIAZINE DRUGS IN AQUEOUS SOLUTION
}

\author{
MUHAMMAD USMAN ${ }^{1,6}$, MUHAMMAD ARIF CHEEMAA, , ABBAS KHAN ${ }^{3,6}$, ZAHOOR HUSSAIN FAROOQI ${ }^{4,6}$, \\ VICTOR MOSQUERA ${ }^{5}$ AND MOHAMMAD SIDDIQ ${ }^{6, *}$
}

\author{
${ }^{1}$ Department of Chemistry, Government College University, Faisalabad, Pakistan \\ ${ }^{2}$ Department of Chemistry, Government College Gujranwala, Pakistan \\ ${ }^{3}$ Department of Chemistry, Abdul Wali Khan University, Mardan, Pakistan \\ ${ }^{4}$ Institute of Chemistry, University of the Punjab, Lahore, Pakistan \\ ${ }^{5}$ Department of Condensed matter Physics, University of Santiago de Compostela, E-15782 Santiago de Compostela, Spain \\ ${ }^{6}$ Department of Chemistry, Quaid-i-Azam University, Islamabad 45320, Pakistan
}

(Received: July 27, 2009 - Accepted: May 31, 2013)

\begin{abstract}
Association characteristics of amphiphilic phenothiazine drugs Fluphenazine and Trifluperazine dihydrochloride in water have been examined and their thermodynamic parameters have been calculated using conductometry. The electrical conductivity was measured as a function of concentration at various temperatures and $\mathrm{cmc}$ was calculated in the temperature range of $20-50^{\circ} \mathrm{C}$. Thermodynamic parameters i.e. standard free energy of micellization, $\Delta G_{m}^{\circ}$, standard enthalpy of micellization, $\Delta H_{m}^{\circ}$ and standard entropy of micellization, $\Delta S_{m}^{\circ}$ were calculated from cmc value using closed association model.
\end{abstract}

\section{INTRODUCTION}

A large number of drugs exhibit colloidal behavior and their properties mainly depend on the nature of aromatic ring system of hydrophobic moiety. They are useful in probing the relationship between molecular structure and physicochemical properties. One of the mechanisms to serve this purpose is to study the thermodynamics of their aggregation and the factors governing this process ${ }^{1}$. The tricyclic antidepressant drugs are the family of structurally related compounds suited ideally for exploration of this relationship. They possess an almost planar ring system with a short hydrocarbon chain carrying a terminal, charged nitrogen atom ${ }^{2}$.

Phenothiazine compounds are commonly used in clinical medicines as antipsychotic and tranquilizing drugs. They have interesting physicochemical properties associated with their ability to self aggregate forming "micelle-like" structure as well as properties to change natural and model biomembranes. Their unusual association characteristics derive from their rigid tricyclic hydrophobic group. The flexibility of hydrophobic group, for example, in the drugs with diphenyl methane structure is conductive to closed or micellar association. However linkage of aromatic rings in the form of rigid, planar nucleus leads to open or continuous association. The rigidity and planarity of aromatic groups, although an important requirement for latter is not the only structural feature influencing the mode of association, playing also alkyl side chain attached to it, a key role. We have already examined thermodynamic properties of Phenothiazine drug thioridazine hydrochloride ${ }^{3}$ butriptyline and doxepine hydrochloride ${ }^{4}$, warfarine sodium salt ${ }^{5}$, promazine and triflupromazine hydrochlorides ${ }^{6}$, certizine hydrochloridel ${ }^{7}$, Dexamethasone Sodium Phosphate and Citalopram Hydrobromide ${ }^{9}$ in aqueous solution.

In this article we intend to discuss the thermodynamic properties of structurally related phenothiazine drugs in aqueous solution which have the following chemical structure.

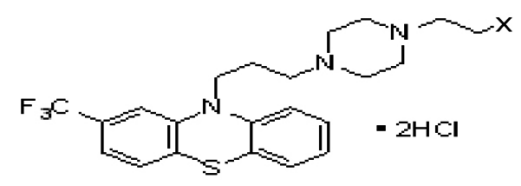

Fluphenazine, $\mathrm{X}=\mathrm{CH}_{2}-\mathrm{CH}_{2}-\mathrm{OH}$

Trifluperazine, $\mathrm{X}=\mathrm{CH}_{3}$

Scheme 1. Chemical structure of Fluphenazine and Trifluperazine dihydrochloride

\section{EXPERIMENTAL}

\section{Reagents}

Fluphenazine dihydrochloride $[\mathrm{C} 22 \mathrm{H} 26 \mathrm{~F} 3 \mathrm{~N} 3 \mathrm{OS} .2 \mathrm{HCl}]$ and trifluperazine dihydrochloride [C21H24F3N3OS.2HCl] with Molar mass $510.5 \mathrm{gmol}^{-1}$ and $480 \mathrm{gmol}^{-1}$ respectively were purchased from Sigma Chemical Co. and were used as received. Solutions were made up by weight at room temperature using a METTLER AT20 balance with a precision of $0.001 \mathrm{mg}$ and double-distilled, deionized water. To avoid concentration gradient, all solutions were stirred before the measurements. All the glassware and Teflon troughs were cleaned using alkaline detergent and rinsed in double distilled water.

\section{Apparatus:}

Specific Electric conductivity measurements: Conductivities were measured with an HP 4285A Precision LCR meter equipped with an HP $5050 \mathrm{~A}$ colloid dielectric probe. The probe is especially designed to measure conductances and to avoid polarization that occur when probe is constructed from place condenser plates. Specific conductivities were measured at (20-50) ${ }^{\circ} \mathrm{C}$ with $10^{\circ}$ increment. The measuring cell was immersed in a thermostat bath keeping the temperature control within $\pm .01^{\circ} \mathrm{C}$, and was calibrated with aqueous solution of $\mathrm{KCl}$ over appropriate concentration range using the molar conductivity data of Schedlovsky ${ }^{10}$ and Chambers et $\mathrm{al}^{11}$.

\section{RESULTS AND DISCUSSION}

The self aggregation study of Fluphenazine and Trifluperazine dihydrochlorides is carried out by a fundamental quantity, the critical micelle concentration, the concentration where micellization just starts. Since this process is very complex hence it is very difficult to pinpoint the concentration where it actually does happen. That's why people are still trying to make an easy way available to determine this concentration which is very important in many biological and industrial phenomenons ${ }^{12}$. The experimental determination of specific conductivities provides an efficient and sensitive technique to detect cmc. It helps to detect second and even third critical concentration (if any). At each temperature concentration dependence of electrical conductivity shows a gradual increase of slope.

The inflection points were made visible by employing Origin program. In agreement with Phillips ${ }^{13}$, the critical micelle concentration is defined as

$$
\left(\frac{d^{3} k}{d c^{3}}\right)=0
$$




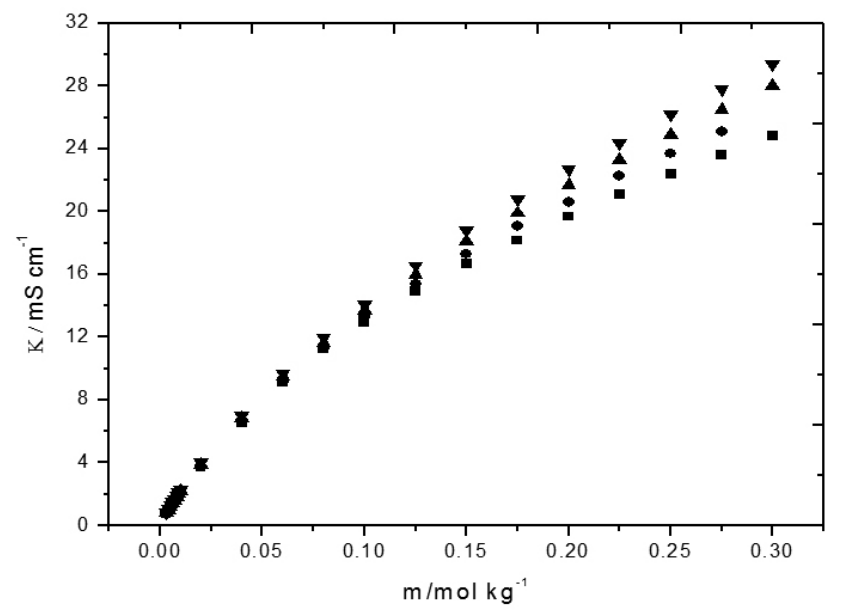

Figure 1. The plot of specific Conductivity $(\mathrm{mS})$ versus molality, $\mathrm{m},(\mathrm{mol} / \mathrm{Kg})$, for aqueous solution of Trifluperazine $2 \mathrm{HCl}$ at $293 \mathrm{~K}(\boldsymbol{\bullet}), 303 \mathrm{~K}(\bullet), 313 \mathrm{~K}(\boldsymbol{\Delta})$ and $323 \mathrm{~K}(\boldsymbol{\nabla})$.

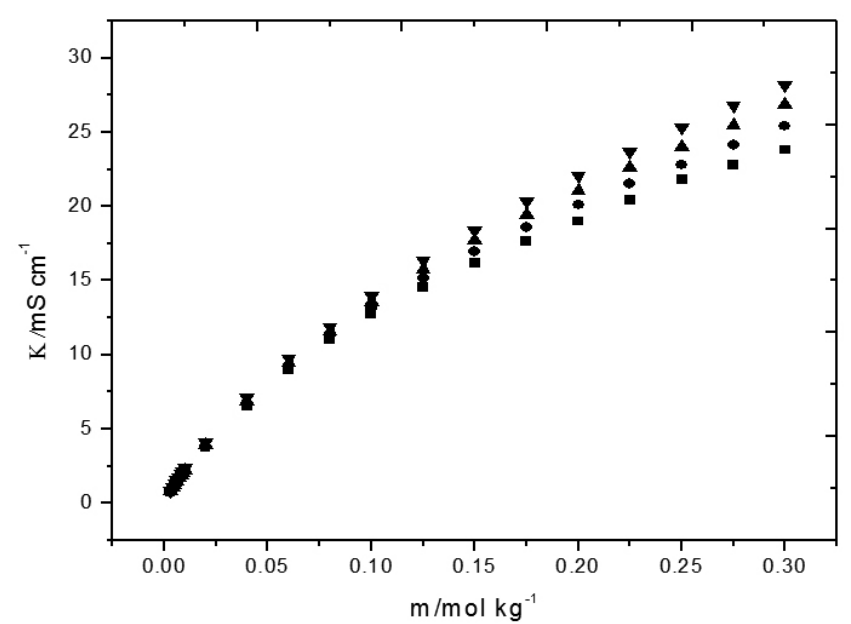

Figure 2. The plot of specific Conductivity $(\mathrm{mS})$ versus molality, $\mathrm{m},(\mathrm{mol} / \mathrm{Kg})$, for aqueous solution of Fluphenazine $2 \mathrm{HCl}$ at $293 \mathrm{~K}(\boldsymbol{\bullet}), 303 \mathrm{~K}(\bullet), 313 \mathrm{~K}(\boldsymbol{\Delta})$ and $323 \mathrm{~K}(\boldsymbol{\nabla})$.

Where $\mathrm{k}$ denotes the conductivity and $\mathrm{c}$ the critical micelle concentration $(\mathrm{cmc})$. The $\mathrm{cmc}$ can also be determined by the intersection of two straight lines of concentration-conductivity plot, above and below the $\mathrm{cmc}$. The precision of measurement depends on the width of concentration range over which the change in physical properties are observed. Two inflection points are obtained in the conductivity plot for both drugs. They are indicated as $\mathrm{cc}_{1}$ and $\mathrm{cc}_{2}$. The $\mathrm{cc}_{1}$ is taken as $\mathrm{cmc}$ because it has reasonable agreement with the value obtained by surface tension data of these drugs ${ }^{14}$. There is again a change in slope of conductivity-concentration plot at $\mathrm{cc}_{2}$ which may be related with structural rearrangement of aggregates previously formed in order to minimize the energy of solution, as occurred for number antidepressant and phenothiazine amphiphilic drugs. Its value doesn't have any clear temperature dependence ${ }^{4}$.

The value of $\mathrm{cmc}$ increases with temperature because the degree of hydrophillic dehydration, at high temperature, is greater than that of hydrophobic dehydration which enhances the repulsion among hydrophilic groups and renders micellization difficult consequently increasing the $\mathrm{cmc}$ value ${ }^{14}$. The $\mathrm{cmc}$ of Trifluperazine $2 \mathrm{HCl}$ has lower value than that of Fluphenazine $2 \mathrm{HCl}$ because the former is more hydrophobic as $-\mathrm{OH}$ group reduces hydrophobicity of Fluphenazine $2 \mathrm{HCl}$.. The value of $\mathrm{cmc}$ helps to calculate value of thermodynamic parameters of drugs i.e. $\Delta G_{m}^{\circ}, \Delta H_{m}^{\circ}$ and $\Delta S_{m}^{\circ}$ These parameters are called standard free energy of micellization, standard enthalpy of micellization and standard entropy of micellization respectively. In this case we are using $\mathrm{cmc}$ in term of mole fraction rather than molality or molarity. We need to define standard state for thermodynamic parameters to be calculated for micellization process. The hypothetical standard state for surfactants in the aqueous phase is taken to be the solvated monomer at unit mole fraction with properties of infinitely dilute solution. For the surfactant in the micellar state, the micellar state is itself considered to be the standard state ${ }^{16}$.

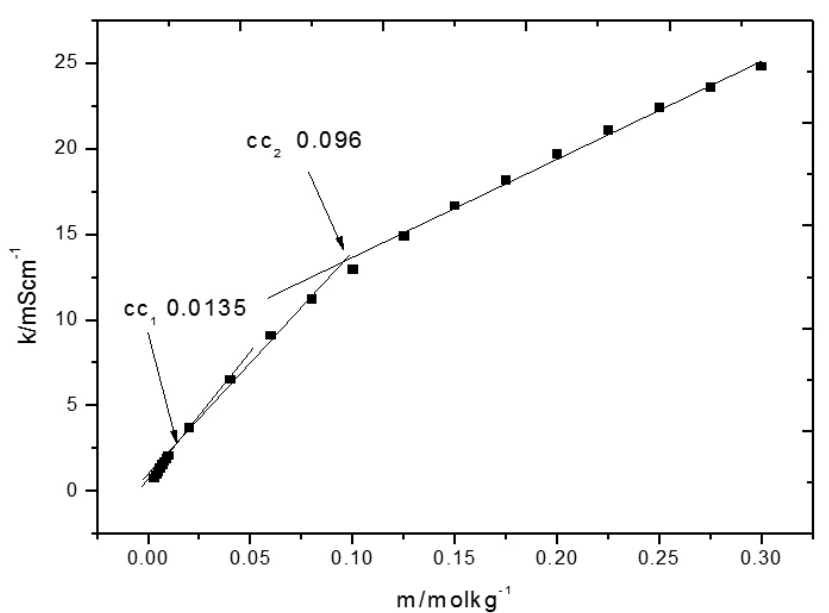

Figure 3. The plot of specific Conductivity $(\mathrm{mS})$ versus molality, $\mathrm{m},(\mathrm{mol} / \mathrm{Kg})$, for aqueous solution of Trifluperazine $2 \mathrm{HCl}$ at $293 \mathrm{~K}$.

The thermodynamic parameters e.g. Gibbs free energy of micellization, $\Delta G_{m}^{\circ}$ was calculated from equation 2, while Enthalpy of micellization, $\Delta H_{m}^{\circ}$ and Entropy of micellization, $\Delta S_{m}^{\circ}$ were calculated from conductivity data using equation 3 and 4 respectively.

$$
\begin{aligned}
& \Delta G_{m}^{0}=(1+\alpha) R T \ln X_{c m c} \\
& \Delta H_{m}^{\circ}=-R T^{2}\left[\frac{\partial(1+\alpha)\left(\ln X_{c m c}\right)}{\partial T}\right]_{P} \\
& \Delta S_{m}^{\circ}=\frac{\Delta H_{m}^{\circ}-\Delta G_{m}^{\circ}}{T}
\end{aligned}
$$

Here $\mathrm{Xcmc}$ is $\mathrm{cmc}$ in term of mole fraction while $\alpha$ is degree of counter ion binding. An approximate value of $\alpha$ is calculated as

$$
\alpha=1-\beta=1-\frac{S_{2}}{S_{1}}
$$

Where $\beta$ is degree of ionization while S2 and S1 are slopes of conductivityconcentration plot after and before $\mathrm{cmc}$ respectively.

Table 3 and 4 show the value of thermodynamic parameters along with the values of $\mathrm{cc} 1, \mathrm{cc} 2$, degree of counter ion binding $(\alpha)$ and degree of ionization $(\beta)$ for both Phenothiazine drugs . 
Table 1: Various parameters calculated from specific conductivity measurements for Fluphenazine $2 \mathrm{HCl}$.

\begin{tabular}{|c|c|c|c|c|c|c|c|}
\hline$\frac{T}{K}$ & $\frac{\mathrm{cmc}}{\mathrm{mmolkg}^{-1}}$ & $\frac{c_{2}}{m^{2}{ }^{2} k^{-I}}$ & $\frac{\Delta H_{m}}{K_{J m o l}}$ & $\frac{\Delta G_{m}}{K J m o l^{-1}}$ & $\frac{\Delta S_{m}}{J K^{-1} m o l^{-1}}$ & $\alpha$ & $\beta$ \\
\hline 293 & $17 \pm 0.65$ & $110 \pm 4.2$ & $-5.60 \pm 0.73$ & $-26.8 \pm 0.84$ & $72.15 \pm 0.95$ & 0.590 & 0.407 \\
\hline 303 & $17.5 \pm 0.35$ & $118 \pm 2.3$ & $-5.98 \pm 0.49$ & $-27.9 \pm 0.51$ & $72.44 \pm 1.03$ & 0.600 & 0.400 \\
\hline 313 & $19.3 \pm 0.2$ & $140 \pm 4.2$ & $-6.38 \pm 0.37$ & $-28.75 \pm 0.39$ & $71.47 \pm 1.01$ & 0.606 & 0.393 \\
\hline 323 & $20.0 \pm 0.31$ & $140 \pm 3.2$ & $-6.80 \pm 0.53$ & $-29.7 \pm 0.47$ & $70.87 \pm 0.93$ & 0.610 & 0.390 \\
\hline
\end{tabular}

Table 2: Various parameters calculated from specific conductivity measurements for Trifluperazine $2 \mathrm{HCl}$.

\begin{tabular}{|c|c|c|c|c|c|c|c|}
\hline$\frac{T}{K}$ & $\frac{\mathrm{cmc}}{m m o l \mathrm{Kg}^{-1}}$ & $\frac{c_{2}}{m m o l \mathrm{Kg}^{-1}}$ & $\frac{\Delta H_{m}}{\mathrm{kjmol}^{-1}}$ & $\frac{\Delta G_{m}}{\mathrm{kjmol}^{-1}}$ & $\frac{\Delta S_{m}}{J K^{-I} \mathrm{~mol}^{-1}}$ & $\alpha$ \\
\hline 293 & $13.5 \pm 0.37$ & $96 \pm 1.52$ & $-20.70 \pm 0.53$ & $-28.24 \pm 0.59$ & $25.70 \pm 0.45$ & 0.618 \\
303 & $14.9 \pm 0.20$ & $109 \pm 2.08$ & $-22.15 \pm 0.31$ & $-29.50 \pm 0.44$ & $24.29 \pm 0.35$ & 0.382 \\
313 & $17.5 \pm 0.45$ & $122 \pm 1.2$ & $-23.64 \pm 0.55$ & $-30.18 \pm 0.59$ & $20.88 \pm 0.51$ & 0.631 & 0.369 \\
323 & $18.4 \pm 0.33$ & $122 \pm 2.1$ & $-25.17 \pm 0.36$ & $-31.33 \pm 0.52$ & $19.07 \pm 0.39$ & $0 . .636$ & 0.364 \\
\hline
\end{tabular}

At higher temperature the value of cmc increases while that of $\Delta \mathrm{Gm}$ becomes more negative. It apparently seems contradicting but actually it is not so because $\Delta \mathrm{G}$ is not only dependent on cmc but also on the degree of counter ion binding and temperature. Published data for a number of amphiphiles makes it clear that process of micellization may be more spontaneous even if $\mathrm{cmc}$ is increasing. The increase in cmc means delay in micellization process and increase in negative value of $\Delta \mathrm{G}$ is indicative of spontaneity of process ${ }^{6}$.

The large negative value of $\Delta G_{m}^{\circ}$ indicates that micellization is a thermodynamically favorable process. "The values of $\Delta G_{m}^{\circ}$ become more negative with temperature showing more spontaneity of process at higher temperature. The positive values of $\Delta S_{m}^{\circ}$ and negative value of $\Delta H^{\circ}$, at each temperature, connotes that micellization is a both entropy and enthalpy driven process ${ }^{15}$ where, in addition to hydrophobic, electrostatic interactions play a vital role. The negative values of $\Delta H^{\circ}$ also let us know about exothermic nature of process. The decrease in value of $\Delta S^{\circ}$ and increase in value of $\Delta H_{m}^{\circ}$ with temperature displays that hydrophobic interactions become weaker while electrostatic ones become stronger. The $\Delta H^{\circ}$ is the sum of change in enthalpies arising from hydrophobic interactions, electrostatic interactions, hydration of polar head groups and counter ion binding to micelles. A negative value of $\Delta H^{\circ}$ may occur when hydration of water molecules around hydrophilic heads groups become more important than destruction of water structure around hydrophobic groups of monomers. The increase in temperature causes dehydration of hydrophilic groups, thus making electrostatic repulsion stronger. The positive values of $\Delta S^{\circ}$ are due to transfer of hydrophobic chains of drugs from aqueous environment to micelle core ${ }^{16}$. It is accepted that in the immediate vicinity of hydrophobic groups there is strengthening of hydrogen bonding between water molecules. This hydration of hydrophobic groups is quite different than the usual solvent-solute interaction and is termed as hydrophobic hydration. The water molecules in neighborhood of hydrophobic groups are more attracted by nearby water molecules. This corresponds to tightening of water structure around hydrophobic groups ${ }^{2}$. A consequence of this situation is that internal torsional vibrations of chains are restricted in solution. The more ordered structure of water molecules around hydrophobic chains and restriction in vibrations of hydrophobic groups leads to decrease in entropy of system. The removal of hydrophobic groups from aqueous environment is entropically favourable leading to disruption of highly organized water structure and removal of mobility constraints on hydrocarbon chain $^{16}$. The dehydration of hydrophobic parts causes a plunge in $\Delta S_{m}^{\circ}$ values with temperature. The comparison of $\Delta G_{m}^{\circ}$ values of both drugs indicates that Trifluperazine $2 \mathrm{HCl}$ is more hydrophobic than Fluphenazine $2 \mathrm{HCl}$ due to presence of different substituents in molecular structure. The micellization of the former is, thus, more spontaneous. These values are in good agreements to those reported for other drugs $s^{4,5,6}$.

\section{CONCLUSION}

The comparative study of thermodynamic properties of Fluphenazine and Trifluperazine dihydrochlorides has been carried out from specific conductivity measurements. The value of $\mathrm{cmc}$ is obtained by employing Origin Program. The value of cmc increases with increase in temperature because at high temperature the degree of hydrophobic dehydration is less than hydrophilic dehydration. This phenomenon opposes micellization and hence increases the cmc. Trifluperazine $2 \mathrm{HCl}$ has lower value of $\mathrm{cmc}$ and more negative value of $\Delta G^{\circ}$ than Fluphenazine $2 \mathrm{HCl}$ because it has more hydrophobic character. The $\Delta G_{m}^{\circ}$ values of both drugs are negative and become more negative at high temperature showing that the process of micellization becomes more spontaneous with temperature. The positive value of $\Delta S_{m}^{\circ}$ and negative value of $\Delta H_{m}^{\circ}$ indicates that micellization is both entropy as well as enthalpy driven and is equally supported by both hydrophobic and electrostatic interactions. The positive value of $\Delta S_{m}^{\circ}$ is due to removal of hydrophobic parts of drugs from aqueous environment to micellar core which destroys ordered water structure around them and enables them to get rid of mobility constraints. The negative value of $\Delta H^{\circ}$ displays that hydration of hydrophilic groups is more important than destruction of water structure around hydrophobic groups.

\section{ACKNOWLEDGMENT}

Muhammad Usman and Abbas Khan wish to acknowledge Higher Education Commission (HEC), Pakistan for the financial support under Indigenous $\mathrm{PhD}$ Scholarship programme.

\section{REFERENCES}

1. P. Taboada, D. Attwood, J. M. Ruso, M. Garcia, V. Mosquera, Langmuir 17,173,(2001).

2. P. Taboada, P.M. Landeira, J.M. Ruso, M. Garcia, V. Mosquera, Colloids and Surfaces A: Physicochemical and Engineering Aspects 197, 95, (2002). 
3. M. A. Cheema, S. Barbosa, P. Taboada, E. Castro, M. Siddiq, V. Mosquera, Chemical Physics, 328, 243,(2006).

4. M. A. Cheema, S. Barbosa, P. Taboada, M. Siddiq, V. Mosquera, Molecular Physics 104, 3203,(2006).

5. M.A. Cheema; P. Taboada; E. Castro, M. Siddiq, V. Mosquera, J. Chem. Eng. Data, 52, 2315,(2007).

6. M. A, Cheema, P. Taboada, E. Castro, M. Siddiq, V. Mosquera, J. Chem. Thermodynamics 40, 298, (2008).

7. M.Usman, A.Khan, M.Siddiq, J.Chem. Soc. Pak 31, 221(2009).

8. A. Shah, A.M. Khan, M.Usman, R.Qureshi, M.Siddiq, S.S.Shah. J. Chil. Chem. Soc. 54, 134 (2009).
9. M.Usman, A.Khan, M.Siddiq, J.Chem. Soc. Pak 32, 1, (2010).

10. T. Shedlovsky, J. Am. Chem. Soc. 54, 1411, (1932)

11. J. F. Chambers, R.H. Stokes, J. Phy. Chem. 60, 985, (1956).

12. A. M. Khan, S.S. Shah, J. Chem. Soc. Pak. 30, 186, (2008).

13. J. N. Phillips, Trans Faraday Soc. 51, 561,(1955).

14. M. A. Cheema; P. Taboada.; S. Barbosa; M. Siddiq; V. Mosquera; J. Chem. Eng. Data 53, 368, (2008).

15. F. Akhtar; M.A. Hoque.; M.A. Khan; J. Chem. Thermodynamics 40, 1082, (2008).

16. D. Attwood, A.T. Florence; Surfactant Systems; Chapman and Hall London: New York 1985. 